\title{
On Feature Combination for Music Classification
}

\author{
Zhouyu Fu, Guojun Lu, Kai-Ming Ting, and Dengsheng Zhang \\ Gippsland School of IT, Monash University, Churchill, VIC 3842, Australia \\ \{zhouyu.fu, guojun. lu , kaiming. ting, dengsheng. zhang\}@infotech . monash . edu . au
}

\begin{abstract}
We address the problem of combining different types of audio features for music classification. Several feature-level and decision-level combination methods have been studied, including kernel methods based on multiple kernel learning, decision level fusion rules and stacked generalization. Eight widely used audio features were examined in the experiments on multi-feature based music classification. Results on benchmark data set have demonstrated the effectiveness of using multiple types of features for music classification and identified the most effective combination method for improving classification performance.
\end{abstract}

\section{Introduction}

Combining multiple features from diverse sources is an effective way to enhance the performance of real-world classification systems. Image classification is one such example that benefited much from feature combination techniques. In recent years, substantial performance gains on challenging benchmark datasets have been reported in the literature [1] by combining multiple features based on different aspects like shape, appearance and texture.

In this paper, we address the problem of using multiple types of features for music classification, which has not yet been adequately investigated in previous studies. Specifically, we have studied a number of candidate schemes for using multiple features, including feature level combination methods such as Multiple Kernel Learning (MKL) for the Support Vector Machine (SVM) classifier [2], and the more general decision level fusion rules such as majority voting, the sum rule 3 and a principled approach to decision fusion called stacked generalization [45]. We have adopted the SVM classifier [6] for both individual feature learning and stacked generalization due to its good classification performance for music classification [7]. Moreover, SVM underlies the inherent formulation of MKL, the feature-level combination method discussed in this paper. Hence, it is best to use SVM for all classification tasks involved to make fair comparison of different combination schemes.

The purpose of this paper is to answer the following three questions regarding feature combination for music classification. Firstly, we are interested in the performance of common individual features for music classification. More importantly, we want to know whether combining multiple features is an effective way to enhance the performance of current music classification systems. Finally, we 
want to identify what is the best feature combination method for the application we study. The answers will be revealed through the empirical evaluation of various feature combination approaches on a benchmark data set.

\section{Audio Features for Music Classification}

Music classification is an emerging area in multimedia and information retrieval. A key problem in music classification is how to efficiently and effectively extract audio features for high level classification. Many types of features have been used in previous study on music classification [8971011, including low-level features such as timbre and temporal features, and mid-level features such as beats and chords.

Low-level features are normally extracted via spectral analysis, and have been used predominantly in music classification systems due to the simple procedures to obtain them and their good performance. There are two types of local features - timbre and temporal features. Both of them are obtained based on spectral analysis of the audio signal. The basic procedures for timbre and temporal feature extraction are quite similar. First, a song is split into small local windows. The truncated signal segment within each local window is assumed to be stationary, a pre-requisite for the application of various spectral analysis techniques. Standard spectral coefficients are then extracted from each local window, include Fast Fourier Transform Coefficients [10, Mel-Frequency Cepstral Coefficients (MFCC) 91011, Amplitude Spectrum Envelop (ASE) 1211, and Octave based Spectral Contrast (OSC) [1311]. Then the coefficients from neighboring local windows are aggregated to produce a single song-level feature. The main difference between timbre and temporal features is in the way local spectral coefficients are aggregated. Timbre features model the distributions of the coefficients, whereas temporal feature modeling treats the coefficients as time series data and concerns their temporal evolution.

Most music classification systems are based on the use of low-level features alone 871011. Alternatively, mid-level features like beats 9 and chords 14 have also been used in some systems to supplement or substitute low-level features. Compared to low-level features, mid-level features can be better interpreted and have more to do with human perception of music. Nevertheless, whether mid-level features are better than low-level features for music classification tasks are still an open question.

Here, our focus is on the combination of different features obtained at different levels to enhance the performance of music classification systems. Each type of feature described above captures some information of music from a different perspective. Hence, they should complement each other for music classification. It is expected that by combining them better classification performance can be achieved. For this purpose, we have used 8 types of individual features in this paper, including three timbre features based on three different spectral coefficients (SMFCC, SASE and SOSC), three temporal features based on the fluctuation pattern [8] of the same three coefficients (TMFCC, TASE and TOSC), as well as 
two mid-level features of beat (B) [9] and chord (C) [14]. These features, either individually or in combination, have been widely used in music classification and provided good empirical classification performance.

It has been shown in [10] and [11 that combining multiple features can improve over the performance of classification using a single feature type. However, both works are limited in the types of features being investigated. Only low-level features were used in [10, and [11] explored the combination of temporal features alone. It is still unclear whether mid-level features are useful for classification or not. Also, as mentioned earlier, we explore the use of multiple different features under the SVM classification framework, which provides quite strong classification at individual feature level. It is thus not evident whether combination yields any further performance improvement. On the other hand, AdaBoost with decision stumps was used in 10 to combine attributes of features. Many weak classifiers were generated in the process and combined by the AdaBoost framework. Later we can see that the performances of [10] and 11] are inferior to ours on feature combination. It is also worth stressing that our problem is different from feature selection. In feature selection, a subset of attributes is selected from all feature attributes that improves over classification based on the full-length feature vector. In the feature combination problem discussed in this paper, we are given multiple feature vectors for each example. The purpose is to find a way to combine the feature vectors to improve the performance over any single feature vector.

\section{Methods for Feature Combination}

We begin with a definition of the feature combination problem. Given a labeled data set $\left\{\left(\left[x_{i}^{1}, \ldots, x_{i}^{M}\right], y_{i}\right)\right\}_{i=1, \ldots, N}$ of size $N$, where $x_{i}^{m} \in \mathbb{R}^{d_{m}}$ is the $m$ th feature vector with feature dimension $d_{m}$ for the $i$ th training instance and $y_{i} \in\{1, \ldots, K\}$ is its class label. The purpose of feature combination is to learn a classification rule $f: \mathcal{X} \rightarrow\{1, \ldots, K\}$ from the $M$ feature vectors with $\mathcal{X} \subset \mathbb{R}^{d_{1}} \times \cdots \times \mathbb{R}^{d_{m}}$. Depending on the level feature combination is performed, feature combination methods can be categorized into two categories. Decisionlevel methods learn a classifier for each individual feature type and combine the output of individual classifiers for label prediction without modifying the feature vectors. Feature-level methods combine the individual feature vectors to form a new feature vector for classification. Next, we discuss various feature combination methods in each of the above two categories. Notice that our discussion is far from comprehensive and has omitted many combination methods proposed in the literature. We have only selected a few typical ones that were well represented and most widely used.

\subsection{Decision-Level Fusion Methods}

In decision level fusion, each individual classifier can return either a single label, a ranking or real-valued output. We assume the latter case that the classifier 
trained on the $m$ th feature outputs an vector $\mathbf{F}\left(x^{m}\right)=\left[f^{1}\left(x^{m}\right), \ldots, f^{K}\left(x^{m}\right)\right] \in$ $\mathbb{R}^{K}$ for a testing instance $x$. Each entry $f^{k}\left(x^{m}\right)$ in the vector indicates the confidence value for the $k$ th class. The larger the value of $f^{k}\left(x^{m}\right)$ relative to the other entries, the more likely that $x$ belongs to class $k$ based on the $m$ th classifier alone. This also includes output labels $\left(f^{k}\left(x^{m}\right)=1\right.$ if $x$ belongs to class $k$ and $f^{k}\left(x^{m}\right)=0$ otherwise) and rankings $\left(\mathbf{F}_{i}\right.$ is a permutation of $\{1, \ldots, K\}$ with $K$ being the top rank and 1 the lowest rank) as special cases. With the above notations, we now present the fusion schemes in below

Majority Voting is the simplest and most widely used decision level fusion rule. The label of testing instance $x$ is given by

$$
\arg \max _{k=1, \ldots, K} \sum_{m=1, \ldots, M} \delta_{k, m}
$$

where $\delta_{k, m}$ is a hard decision function which equals 1 if and only if the $m$ th classifier votes for class $k$, that is, $f^{k}\left(x^{m}\right)$ is larger than other $f^{j}\left(x^{m}\right)$ 's for $j \neq k$. Despite its simplicity, majority voting ignores the values of classifier output which encode confidence levels on prediction. To fix it, an alternative fusion rule like the sum rule can be used.

Sum Rule uses decision values $f^{k}\left(x^{m}\right)$ 's directly in aggregation

$$
\arg \max _{k=1, \ldots, K} \sum_{m=1, \ldots, M} f^{k}\left(x^{m}\right)
$$

Hence the larger $f^{k}\left(x^{m}\right)$ is, the more it contributes to the final score for class $k$.

Besides majority voting and sum rules, a number of alternative rules can be used with simple algebra operations. The discussion can be found in the overview paper [3]. A probabilistic framework is also developed in [3] that incorporates all fusion rules as special cases. In the probabilistic framework, $f^{k}\left(x^{m}\right)$ 's become the posterior probabilities. However, to use the above two fusion rules, we only have to assume that classifier output $f^{k}\left(x^{m}\right)$ 's are proportional to the posterior probabilities, instead of requiring them to be probabilistic output. This is a reasonable assumption since larger value of $f^{k}\left(x^{m}\right)$ indicates a higher likelihood of class $k$. Thus we can generalize these rules to take real-valued scores.

\section{Stacked Generalization}

The above fusion rules are defined in an unsupervised fashion without using the label information in the training data. Stacked generalization 4 . provides a principled framework for learning supervised decision rules. It treats the output values $f^{k}\left(x^{m}\right)$ 's returned by individual classifiers as new features that can be used for classification. Specifically, it creates the following feature map for training instance $x_{i}$

$$
\mathbf{F}_{i}=\left[f^{1}\left(x_{i}^{1}\right), \ldots, f^{K}\left(x_{i}^{1}\right), \ldots, f^{1}\left(x_{i}^{M}\right), \ldots, f^{K}\left(x_{i}^{M}\right)\right] \in \mathbb{R}^{K M}
$$

where the first $K$ feature elements are taken from decision values returned by the first classifier, the next $K$ feature elements are values returned by the second classifier, and so on. The total feature dimension is $K M$ for $K$-class classification 
with $M$ features. A new training set can then be constructed $\left\{\left(\mathbf{F}_{i}, y_{i}\right)\right\}_{i=1, \ldots, N}$ on top of which a new classifier is learned. Any forms of classifier can be used for stacked generalization but linear classifiers are preferred due to its efficiency and performance [5].

Here, we adopted the linear SVM classifier which, in the binary case, can be learned by solving the following optimization problem

$$
\min _{\mathbf{w}, b} \frac{1}{2}\|\mathbf{w}\|^{2}+\lambda \sum_{i=1}^{N} \max \left(0,1-y_{i}\left(\left\langle\mathbf{w}, \mathbf{F}_{i}\right\rangle+b\right)\right)
$$

where $y_{i} \in\{1,-1\}$ denotes the binary class label for the $i$ th instance, $\mathbf{w}$ and $b$ are the weight and bias of the linear discriminant function. In the above equation, the first term is the regularization term, whereas the second term specifies a Hinge loss on misclassification. $\lambda$ controls the trade-off between the two terms.

The SVM is best solved in its dual form in the following

$$
\max _{0 \leq \alpha \leq \lambda} \sum_{i=1}^{N} \alpha_{i}-\frac{1}{2} \sum_{i=1}^{N} \sum_{j=1}^{N} \alpha_{i} y_{i} \alpha_{j} y_{j} K\left(\mathbf{F}_{i}, \mathbf{F}_{j}\right) \quad \text { s.t. } \quad \sum_{i} \alpha_{i} y_{i}=0
$$

where $\alpha_{i}$ 's are dual variables and $K\left(\mathbf{F}_{i}, \mathbf{F}_{j}\right)=\left\langle\mathbf{F}_{i}, \mathbf{F}_{j}\right\rangle$ is the kernel function defined as the inner product between two feature vectors. Multi-class classification problems are tackled with a one-vs-all strategy by training $K$ classifiers to differentiate between class $k$ and non-class $k$ for $k=1, \ldots, K$. A testing instance is assigned to the class with the largest output value.

\subsection{Feature-Level Combination Methods}

Feature-level combination methods are usually developed under specific classification framework. Here we focus on feature-level combination with the SVM classifier. Despite the simplicity of linear SVM, it can not handle nonlinear data. The nonlinear SVM classifier is usually used instead for real-world classification problems. This is achieved by utilizing the kernel trick. Specifically, let $\phi$ define a nonlinear feature mapping for feature vector $\mathbf{F}_{i}$. The explicit form of $\phi\left(\mathbf{F}_{i}\right)$ is unknown, but the inner product between two nonlinear features is well defined by the kernel function $K\left(\mathbf{F}_{i}, \mathbf{F}_{j}\right)=\left\langle\phi\left(\mathbf{F}_{i}\right), \phi\left(\mathbf{F}_{j}\right)\right\rangle$. In this case, we can solve the dual formulation in Equation 4 by plugging into a different kernel function. Common nonlinear kernels include Gaussian, polynomial and sigmoid kernels.

Feature Concatenation is the most straightforward feature-level operation to form a composite feature by concatenating all individual features. The composite feature is a long feature vector given by $\mathbf{x}=\left[\mathbf{x}^{1}, \ldots, \mathbf{x}^{M}\right]$, which can be used for feature classification. In the case of SVM, it is used for computing the new kernel $K\left(\mathbf{x}_{i}, \mathbf{x}_{j}\right)$.

\section{Kernel Averaging}

Alternatively, we can do feature concatenation in the implicit feature space given by the mapping function $\phi$. This is equivalent to averaging the kernels induced 
by the individual feature maps. Specifically, the concatenated nonlinear feature is given by $\phi(\mathbf{x})=\frac{1}{\sqrt{M}}\left[\phi\left(\mathbf{x}^{1}\right), \ldots, \phi\left(\mathbf{x}^{M}\right)\right]$. This is equivalent to defining a composite kernel function

$$
K\left(\mathbf{x}_{i}, \mathbf{x}_{j}\right)=\left\langle\phi\left(\mathbf{x}_{i}\right), \phi\left(\mathbf{x}_{j}\right)\right\rangle=\frac{1}{M} \sum_{m=1}^{M}\left\langle\phi\left(\mathbf{x}_{i}^{m}\right), \phi\left(\mathbf{x}_{j}^{m}\right)\right\rangle=\frac{1}{M} \sum_{m=1}^{M} K\left(\mathbf{x}_{i}^{m}, \mathbf{x}_{j}^{m}\right)
$$

The composite kernel is the average of all individual kernels. We can solve the SVM dual formulation using the composite kernel.

\section{Multiple Kernel Learning (MKL)}

Instead of using uniform weights for the composite kernel in Equation5, a more general formulation is introduced in [2] for learning kernel weights based on the SVM dual formulation,

$$
\begin{gathered}
\min _{\beta \geq 0} \max _{0 \leq \alpha \leq \lambda} \sum_{i=1}^{N} \alpha_{i}-\sum_{i=1}^{N} \sum_{j=1}^{N} \alpha_{i} y_{i} \alpha_{j} y_{j} K_{\beta}\left(\mathbf{x}_{i}, \mathbf{x}_{j}\right) \\
\text { with } \quad K_{\beta}\left(\mathbf{x}_{i}, \mathbf{x}_{j}\right)=\sum_{m=1}^{M} \beta_{m} K\left(\mathbf{x}_{i}^{m}, \mathbf{x}_{j}^{m}\right) \\
\text { s.t. } \quad \sum_{i} \alpha_{i} y_{i}=0 \quad \text { and } \quad \sum_{m} \beta_{m}=1
\end{gathered}
$$

The above problem is generally referred to as multiple kernel learning [2]. The objective function is still convex and hence can be minimized effectively. The solution provides both a set of feature kernel weights as well as the dual variables used to define the nonlinear decision function $f(\mathbf{x})=\sum_{i} \alpha_{i} y_{i} K_{\beta}\left(\mathbf{x}_{i}, \mathbf{x}\right)+b$. For multiclass classification, we learn kernel weights jointly for all classes by taking the sum of objective functions for each class and fixing $\beta$ values in Equation 6 .

\subsection{Recursive Feature Elimination}

Inspired by the idea of [15, we develop a procedure for recursively eliminating redundant features based on stacked generalization. Let $w_{k, m}^{j}$ be the weight of the $j$ th linear SVM for decision value $f^{k}\left(x^{m}\right)$, we then define the relevance of the $m$ th feature by the measure $\sum_{j=1}^{K} \sum_{k=1}^{K}\left(w_{k, m}^{j}\right)^{2}$. The larger the relevance, the more useful the feature is for classification. Starting with the full set of features, we can then take the following steps for recursive feature elimination

1. Learn the feature combination model with the remaining features

2. Eliminate the feature with the lowest relevance

3. Repeat the above two steps until the desired number of features is reached

Through the recursive feature elimination procedure, we can determine the importance of each individual feature for classification and retain a subset of features for combination. It also produces a ranking for the individual features based on the order they are eliminated. From the ranking, we can determine the relative importance of individual features. 


\section{Experimental Results}

In this section, we perform feature combination experiments on a benchmark data set for music genre classification. We used the GTZAN data set [9], which contains 1000 song segments in 30 seconds of duration uniformly distributed from 10 genres. For each song, we have extracted eight individual features as described in Section 2. Each classification experiment was repeated 20 times with different random partitioning of training and testing data. For each round, half of the examples in the data set were randomly selected for training and the remaining for testing. All features were examined on the same training and testing set in each round. The LibSVM packag 11 and the Gaussian kernel was used for SVM training. To reduce the scaling effect, each feature attribute has been scaled to zero mean and unit standard deviation for kernel computation. We have also normalized the kernel matrix to unit mean so as to reduce the scaling effect at kernel level. SVM and kernel parameters were chosen via 3 fold cross validation on the training data.

First, we examine the effectiveness of each individual feature set for music genre classification using the SVM classifier. Table 1 shows the average accuracy rates over 20 rounds achieved by individual features for i) each genre class by treating the target genre as the positive class and the other genres as the negative class; and ii) the 10-class problem by classifying each song into one of the 10 genre classes. Accuracy rates for the top performing features are highlighted in bold for each genre and the 10-class problem. These include the feature type with the highest average accuracy and other features with close performances. That is, the differences in accuracy rates between those features and the top feature are not statistically significant based on the outcomes of paired t-tests within $95 \%$ of confidence interval. From the results in Table 11 we can see that chord feature is the best individual feature type for genre classification achieving top performances for 6 out of 10 genres as well as the 10-class problem. Most of the other features obtain similar classification performance except the beat feature, which has significantly lower accuracy rates. However, although being ineffective in overall, beat is best in identifying the disco. This is consistent with our perception of the disco, which is distinguished by its faster rhythm and more frequent beats compared to other music genres.

We now turn our attention to feature combination. We compare the various feature combination schemes discussed in Section 3 including three feature-level combination methods ("FC" for feature concatenation, "AvgK" for kernel average and "MKL" for multiple kernel learning) and three decision-level schemes ("Vote" for majority vote, "Sum" for sum rule, "SG" for stacked generalization). The same setup was adopted from the previous experiment with the same training and testing set partitions and tools for SVM training. The output values of SVM classifiers over individual features were directly used by Vote, Sum and SG with optimal parameters selected from cross validation. The regularization parameter for $\mathrm{SG}$ was also chosen from cross validation. Table 2 shows the

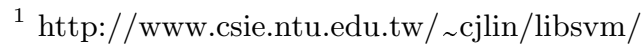


Table 1. Accuracy rates in percentage for individual feature sets

\begin{tabular}{|c|c|c|c|c|c|c|c|c|}
\hline & SMFCC & SASE & SOSC & TMFCC & TASE & TOSC & Beat & Chord \\
\hline \hline Blues & 75.90 & 64.40 & 76.90 & 73.20 & 72.00 & 78.80 & 18.60 & $\mathbf{8 3 . 2 0}$ \\
\hline Classical & 93.00 & 91.50 & $\mathbf{9 4 . 8 0}$ & $\mathbf{9 5 . 8 0}$ & 92.10 & $\mathbf{9 4 . 3 0}$ & 29.40 & 90.20 \\
\hline Country & 68.20 & 72.20 & $\mathbf{7 6 . 1 0}$ & 72.70 & 71.20 & $\mathbf{7 5 . 5 0}$ & 17.70 & 69.90 \\
\hline Disco & 63.30 & 56.60 & 63.00 & 63.20 & $\mathbf{6 9 . 1 0}$ & 66.20 & $\mathbf{7 1 . 6 0}$ & 54.10 \\
\hline Hiphop & 68.90 & 65.20 & 72.40 & 73.80 & 77.50 & 74.90 & 27.10 & $\mathbf{9 6 . 6 0}$ \\
\hline Jazz & 82.40 & 82.50 & 83.40 & 87.80 & 80.10 & 80.00 & 16.00 & $\mathbf{9 8 . 7 0}$ \\
\hline Pop & $\mathbf{9 0 . 0 0}$ & 87.90 & 86.70 & $\mathbf{8 9 . 4 0}$ & 87.20 & 85.40 & 19.40 & 65.00 \\
\hline Metal & $\mathbf{7 7 . 1 0}$ & $\mathbf{7 5 . 6 0}$ & 71.40 & 66.10 & 71.80 & $\mathbf{7 4 . 8 0}$ & 17.90 & $\mathbf{7 7 . 9 0}$ \\
\hline Reggae & 63.80 & 60.40 & 65.90 & 67.60 & 65.90 & 67.60 & 18.10 & $\mathbf{8 1 . 3 0}$ \\
\hline Rock & 52.90 & 23.70 & 40.40 & 48.50 & 45.10 & 52.50 & 10.80 & $\mathbf{7 2 . 3 0}$ \\
\hline 10-class & 73.55 & 68.00 & 73.10 & 73.81 & 73.20 & 75.00 & 24.66 & $\mathbf{7 8 . 9 2}$ \\
\hline
\end{tabular}

Table 2. Accuracy rates in percentage for various feature combination methods

\begin{tabular}{|c|c|c|c|c|c|c|c|}
\hline & Best & Vote & FC & AvgK & Sum & MKL & SG \\
\hline \hline Blues & 83.20 & 86.30 & $\mathbf{8 9 . 6 0}$ & $\mathbf{9 4 . 2 0}$ & $\mathbf{9 1 . 7 0}$ & $\mathbf{9 3 . 7 0}$ & $\mathbf{9 5 . 7 0}$ \\
\hline Classical & 95.80 & 96.80 & 97.00 & 97.20 & 96.60 & 97.50 & 97.00 \\
\hline Country & 76.10 & $\mathbf{8 2 . 9 0}$ & $\mathbf{8 3 . 6 0}$ & $\mathbf{8 8 . 5 0}$ & $\mathbf{8 5 . 5 0}$ & $\mathbf{8 9 . 4 0}$ & $\mathbf{8 9 . 4 0}$ \\
\hline Disco & 71.60 & $\mathbf{7 7 . 6 0}$ & $\mathbf{8 3 . 0 0}$ & $\mathbf{8 3 . 7 0}$ & $\mathbf{8 6 . 1 0}$ & $\mathbf{8 6 . 3 0}$ & $\mathbf{8 6 . 6 0}$ \\
\hline Hiphop & 96.60 & 85.90 & 86.60 & 91.90 & 93.40 & 93.00 & 93.30 \\
\hline Jazz & 98.70 & 92.00 & 91.10 & 96.30 & 98.60 & 97.90 & 98.40 \\
\hline Pop & 90.00 & 91.10 & 90.40 & $\mathbf{9 2 . 5 0}$ & $\mathbf{9 2 . 3 0}$ & $\mathbf{9 3 . 8 0}$ & $\mathbf{9 6 . 3 0}$ \\
\hline Metal & 77.90 & 78.60 & $\mathbf{8 0 . 6 0}$ & $\mathbf{8 8 . 0 0}$ & $\mathbf{8 7 . 8 0}$ & $\mathbf{8 9 . 7 0}$ & $\mathbf{8 7 . 8 0}$ \\
\hline Reggae & 81.30 & 82.90 & 75.10 & $\mathbf{8 4 . 6 0}$ & $\mathbf{8 7 . 5 0}$ & $\mathbf{8 6 . 5 0}$ & $\mathbf{8 5 . 1 0}$ \\
\hline Rock & 72.30 & 68.80 & 70.50 & 73.90 & $\mathbf{7 8 . 5 0}$ & $\mathbf{7 6 . 0 0}$ & $\mathbf{7 8 . 9 0}$ \\
\hline 10-class & 78.92 & $\mathbf{8 4 . 2 9}$ & $\mathbf{8 4 . 7 5}$ & $\mathbf{8 9 . 0 8}$ & $\mathbf{8 9 . 8 0}$ & $\mathbf{9 0 . 3 8}$ & $\mathbf{9 0 . 8 5}$ \\
\hline
\end{tabular}

average accuracy rates achieved by different feature combination methods over 20 rounds for each music genre. For comparison purpose, we have also included the best results returned by the optimal individual feature in the table.

We have highlighted in bold the accuracy rates for combination schemes that outperform the best individual feature in each row of Table 2, This is again determined by comparing the differences in their accuracy rates using paired t-tests. It can be seen from Table 2 that feature combination can much improve the performance of music classification, regardless of the specific combination method being used. Even simple fusion rule like majority voting performs significantly better than the top individual feature for the 10-class problem. Feature combination is effective for 7 out of 10 genres with improved accuracy rates, and the improvement is more evident for those genres that no individual feature can do very well, like disco and country music. The columns of Table 2 are ranked by classification performances for the 10-class problem, with increasing average accuracy rates from left to right. Among the top four combination schemes, 


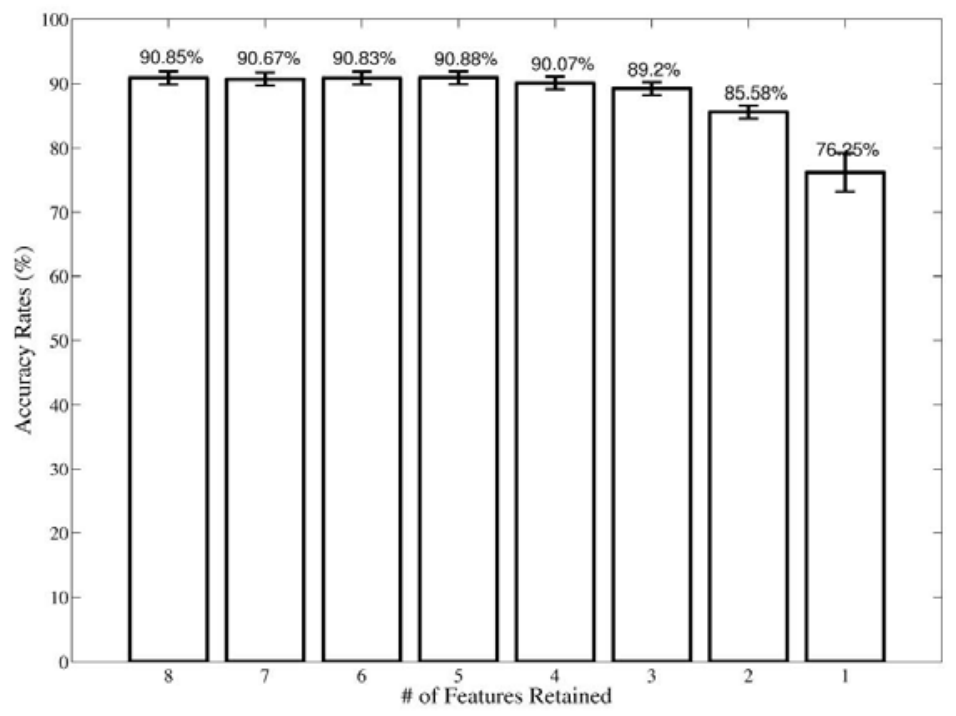

Fig. 1. Results of recursive feature elimination for classification

which achieve significantly better performances than others, decision level fusion schemes (SG and sum rule) perform slightly better than feature level schemes (MKL and average kernel) in overall. Supervised combination schemes (SG and MKL) also outperform their unsupervised counterparts (sum rule and average kernel). Significance tests on the 10-class accuracy rates further corroborates our findings, showing that the differences in accuracy rates obtained by any pair of methods are statistically significant, except for SG versus MKL and MKL versus sum rule.

It is worth mentioning that the best 10-class accuracy rate of $90.9 \%$ achieved by SG outperforms the state-of-the-art genre classification results reported in [10] $(83 \%)$ and [1] $(79.6 \%$ 2), while both of them adopted a multiple feature approach albeit with weaker learners on individual features. This empirically justifies the strength of feature combination with a strong classifier like SVM.

Finally, we examine the relative importance of individual features by applying the recursive feature elimination procedure to the eight audio features using stacked generalization. Figure 1 shows the bar plot of accuracy rates varied against the number of features retained. The error bars in the plot represent the standard deviation over 20 rounds. It can be clearly seen that the accuracy rates are quite consistent with the elimination of the least relevant features. The feature elimination scheme also provides feature ranking results. Ranks returned by different testing rounds are different, but an overall ranking can be determined

${ }^{2}$ The accuracy rate of $91 \%$ reported in [1] is based on 10 -fold cross validation. The result here is based on our implementation of the algorithm and tested for $50 \%-50 \%$ split of training/testing data. 
by sorting the average rank order. From the overall ranking, we find that beat is the weakest individual feature and always the first one to be eliminated, whereas chord is the strongest feature and usually the last one to remain.

\section{Conclusions}

We have studied the problem of multiple feature combination for music classification. Empirical validation showed that the classification performance is much improved by using multiple features at different levels regardless of the combination schemes adopted. Moreover, we have also identified chord as the single best feature for music genre classification and stacked generalization as the optimal combination scheme for multiple feature combination.

\section{References}

1. Varma, M., Ray, D.: Learning the discriminative power invariance trade-off. In: Intl. Conf. on Computer Vision (2007)

2. Lanckriet, G., Cristianini, N., Bartlett, P., Ghaoui, L.E., Jordan, M.I.: Learning the kernel matrix with semidefinite programming. Journal of Machine Learning Research 5, 27-72 (2004)

3. Kittler, J., Hatef, M., Duin, R., Matas, J.: On combining classifiers. IEEE Transactions on Pattern Analysis and Machine Intelligence 20(3), 226-239 (1998)

4. Wolpert, D.: Stacked generalization. Neural Networks 5(2), 241-259 (1992)

5. Ting, K.M., Witten, I.: Issues in stacked generalization. Journal of Artificial Intelligence Research 10, 271-289 (1999)

6. Boser, B.E., Guyon, I., Vapnik, V.: A training algorithm for optimal margin classifiers. In: ACM Conf. Computational Learning Theory, pp. 144-152 (1992)

7. Mandel, M., Ellis, D.: Song-level features and svms for music classification. In: Intl. Conf. Music Information Retrieval (2005)

8. Pampalk, E., Rauber, A., Merkl, D.: Content-based organization and visualization of music archives. In: ACM Multimedia (2002)

9. Tzanetakis, G., Cook, P.: Musical genre classification of audio signals. IEEE Trans. Speech and Audio Processing 10(5), 293-302 (2002)

10. Bergstra, J., Casagrande, N., Erhan, D., Eck, D., Kegl, B.: Aggregate features and ada boost for music classification. Machine Learning 65(2-3), 473-484 (2006)

11. Lee, C.H., Shih, J.L., Yu, K.M., Lin, H.S.: Automatic music genre classification based on modulation spectral analysis of spectral and cepstral features. IEEE Trans. Multimedia 11(4), 670-682 (2009)

12. Kim, H.G., Moreau, N., Sikora, T.: Audio classification based on mpeg-7 spectral basis representation. IEEE Trans. Circuits and Systems for Video Technology $14(5), 716-725(2004)$

13. Lu, L., Liu, D., Zhang, H.J.: Automatic mood detection and tracking of music audio signals. IEEE Trans. Speech and Audio Processing 14(1), 5-18 (2006)

14. Cheng, H.T., Yang, Y.H., Lin, Y.C., Liao, I.B., Chen, H.H.: Automatic chord recognition for music classification and retrieval. In: Intl. Conf. Multi. Expo. (2008)

15. Guyon, I., Weston, J., Barnhill, S., Vapnik, V.: Gene selection for cancer classification using support vector machines. Machine Learning 46(1-3), 389-422 (2002) 